\title{
From Majorana fermions to topological order
}

\author{
Barbara M. Terhal, ${ }^{1}$ Fabian Hassler, ${ }^{1}$ and David P. DiVincenzo ${ }^{1,2}$ \\ ${ }^{1}$ Institute for Quantum Information, RWTH Aachen University, 52056 Aachen, Germany \\ ${ }^{2}$ Department of Theoretical Nanoelectronics, PGI, Forschungszentrum Jülich, 52425 Jülich, Germany
}

(Received 23 January 2012; published 28 June 2012)

\begin{abstract}
We consider a system consisting of a 2D network of links between Majorana fermions on superconducting islands. We show that the fermionic Hamiltonian modeling this system is topologically ordered in a region of parameter space: we show that Kitaev's toric code emerges in fourth-order perturbation theory. By using a Jordan-Wigner transformation we can map the model onto a family of signed 2D Ising models in a transverse field where the signs, ferromagnetic or antiferromagnetic, are determined by additional gauge bits. Our mapping allows an understanding of the nonperturbative regime and the phase transition to a nontopological phase. We discuss the physics behind a possible implementation of this model and argue how it can be used for topological quantum computation by adiabatic changes in the Hamiltonian.
\end{abstract}

DOI: 10.1103/PhysRevLett.108.260504

PACS numbers: 03.67.Lx, 03.65.Vf

Kitaev's well-known toric code $[1,2]$ is a toy model Hamiltonian which demonstrates the concept of topological order in two dimensions; it features a ground space of degeneracy 4 that is topologically protected from sufficiently small perturbations. Thus one can imagine storing a qubit in this ground space of this model such that, at low temperature $T$ compared to the gap, dephasing of such qubit is exponentially suppressed with growing lattice size. More general constructions allow the encoding of many qubits in the ground space and the topological implementation of a CNOT and Hadamard gate by means of Hamiltonian or code deformation [3-6]. In the theory of quantum error correction, this surface code architecture has emerged [7] as one of the more plausible routes to fault-tolerant quantum computation.

In this Letter, we investigate how one could arrive at the toric code Hamiltonian starting from a realistic model of interacting fermions. Kitaev has shown how the toric code emerges in fourth-order perturbation theory from the socalled honeycomb model [8] (see also [9]). As in this previous work, our Hamiltonian has many conserved quantities which we use to understand the stability of the topological phase. Given the recent interest in making proximity-coupled semiconducting nanowires which support weakly interacting Majorana bound states at their ends [10-16], we believe that our model may provide a viable route to the realization of topological quantum computation. Our model may have many other implementations where superconductivity allows for the presence of weak Majorana fermion interactions. The interest in Majorana fermion wires is partially motivated by their fermionicparity protected ground space degeneracy which allows parity protected quantum computation [17-19] and braiding in networks of nanowires [20-23]. The advantage of the approach advocated in this Letter is that the protection is fully topological and no longer based on fermionic-parity conservation. The idea of engineering a topologically ordered Hamiltonian using Josephsonjunction arrays has been explored mostly in the work of Ioffe and co-workers, see, e.g., [24]. We will first discuss the fermionic model and its analysis, then we physically motivate the model and briefly review what would be required for doing topological quantum computation.

We consider the following fermionic Hamiltonian $H=H_{0}+V$, where $H_{0}=\sum_{i}^{2 L^{2}} H_{0}^{i}$ and $i$ labels the square islands in Fig. 1. For simplicity in the analysis, the lattice in Fig. 1(a) has periodic boundary conditions in both directions (see Supplemental Material [25] for a discussion of the model with open boundaries). Each $H_{0}^{i}$ acts on two fermionic modes or four Majorana modes as

$$
H_{0}^{i}=-\Delta c_{a}^{i} c_{b}^{i} c_{c}^{i} c_{d}^{i}
$$

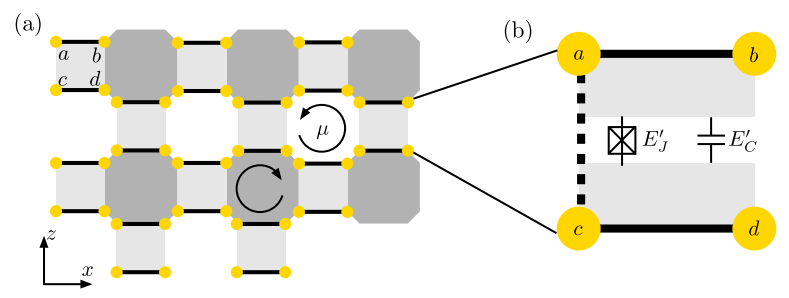

FIG. 1 (color online). (a) Fermionic model studied in this Letter. Each island (light gray square) has four Majorana fermions (yellow dots) labeled as $a, b, c, d$. Fifty percent occupancy is favored for these two fermionic modes, as expressed by the parity constraint in Eq. (1). A weaker quadratic interaction exists between Majorana fermions on islands $i$ and $j$ along diagonal links $V_{i=\mu \pm \hat{z}, j=\mu \pm \hat{x}}$, Eq. (2). Periodic boundary conditions are assumed. (b) Zoomed view of a single island. The Majorana wire has a $C$ shape (black line) in order to be able to tune the overlap between the $a$ - and $c$-Majorana and thus implement an $X$ gate. A $Z$ gate/measurement is implemented by increasing the ratio of $E_{C}^{\prime} / E_{J}^{\prime}$, see main text. 
Further, we have $V=\lambda \sum_{i<j} V_{i, j}$ (with $\lambda>0$ ), where $i, j$ represents the interaction between two Majorana fermions on adjacent islands $i$ and $j$, i.e., $V_{i=\mu \pm \hat{z}, j=\mu \pm \hat{x}}$ for a plaquette $\mu$ equals

$V_{\mu+\hat{z}, \mu-\hat{x}}= \pm i c_{b}^{\mu-\hat{x}} c_{c}^{\mu+\hat{z}}, \quad V_{\mu-\hat{z}, \mu-\hat{x}}= \pm i c_{a}^{\mu-\hat{z}} c_{d}^{\mu-\hat{x}}$,

$V_{\mu-\hat{z}, \mu+\hat{x}}= \pm i c_{c}^{\mu+\hat{x}} c_{b}^{\mu-\hat{z}}, \quad V_{\mu+\hat{z}, \mu+\hat{x}}= \pm i c_{d}^{\mu+\hat{z}} c_{a}^{\mu+\hat{x}}$.

All link operators $V_{\mu \pm \hat{z}, \mu \pm \hat{x}}$ mutually commute. The \pm signs of these terms will be fixed according to the consistent orientation of the plaquettes in Fig. 1; i.e., the link on the top left of a white plaquette $\mu$ represents the interaction $V_{\mu+\hat{z}, \mu-\hat{x}}=i c_{b}^{\mu-\hat{x}} c_{c}^{\mu+\hat{z}}$. Physically, these signs depend on microscopic detail and thus we assume them to be random but fixed. We can find an extensive set of operators which commute with all terms of $H$ and which all mutually commute. These are, first of all, weight- 8 fermionic plaquette operators $\left\{C_{\mu}^{g}, C_{\mu}^{w}\right\}$ [where $g(w)$ stands for gray (white) plaquettes $\mu$ ] which are the product of four link operators around a plaquette

$$
C_{\mu}^{g / w}=c_{d}^{\mu+\hat{z}} c_{a}^{\mu+\hat{x}} c_{c}^{\mu+\hat{x}} c_{b}^{\mu-\hat{z}} c_{a}^{\mu-\hat{z}} c_{d}^{\mu-\hat{x}} c_{b}^{\mu-\hat{x}} c_{c}^{\mu+\hat{z}} .
$$

Second, the torus has two homologically nontrivial closed loops $\gamma_{1}, \gamma_{2}$ and the loop operators $C_{\gamma_{1 / 2}}=$ $\Pi_{(i, j) \in \gamma_{1 / 2}} V_{i, j}$ commute with all link $V_{i, j}$ and island operators $H_{0}^{i}$. Thus, the Hamiltonian is block diagonal with respect to subspaces (sectors) characterized by the eigenvalues $C_{\mu}^{g / w}= \pm 1$ and $C_{\gamma_{1 / 2}}= \pm 1$.

We can first analyze the model in the perturbative regime where $\Delta \gg \lambda$, see, e.g., [8]. The ground space of $H$ at $\lambda=0$ is characterized by $\left\{c_{a}^{i} c_{b}^{i} c_{c}^{i} c_{d}^{i}=+1\right\}$, and thus the ground space on each island is a two-dimensional subspace, a qubit. By convention one can define the logical $X$ and $Z$ operators on this island qubit as

$$
X_{i}=i c_{c}^{i} c_{a}^{i} \equiv i c_{d}^{i} c_{b}^{i}, \quad Z_{i}=i c_{c}^{i} c_{d}^{i} \equiv i c_{b}^{i} c_{a}^{i} .
$$

Let $P_{-}=2^{-2 L^{2}} \Pi_{i=1}^{2 L^{2}}\left(I+c_{a}^{i} c_{b}^{i} c_{c}^{i} c_{d}^{i}\right)$ be the projector onto this $2^{2 L^{2}}$-dimensional unperturbed ground space. The first nontrivial term in the perturbative expansion occurs in fourth order (see Supplemental Material [25]), i.e.,

$$
H_{\mathrm{eff}}=-\frac{5 \lambda^{4}}{16 \Delta^{3}} \sum_{\mu=1}^{2 L^{2}} A_{\mu}+O\left(\frac{\lambda^{6}}{\Delta^{5}}\right),
$$

where $A_{\mu}=Z_{\mu+\hat{z}} X_{\mu+\hat{x}} Z_{\mu-\hat{z}} X_{\mu-\hat{x}}$, i.e., the plaquette terms of the toric code in Fig. 2 [26]. Note that $P_{-} C_{\mu}^{g / w} P_{-}=A_{\mu}^{g / w}$ and hence the four-dimensional toric code ground space of $H$ when $\Delta \gg \lambda$ lies in the $\left\{C_{\mu}^{g / w}=+1\right\}$ sector.

It is important to understand how the topological phase extends to the regime where $\frac{\Delta}{\lambda}=O(1)$, since the gap $\Delta_{\text {eff }}=\frac{20 \lambda}{16}\left(\frac{\lambda}{\Delta}\right)^{3}$ for $\Delta \gg \lambda$ is likely to be quite small compared to the temperature $T$. We can get some insight for this regime by considering higher-order terms in the

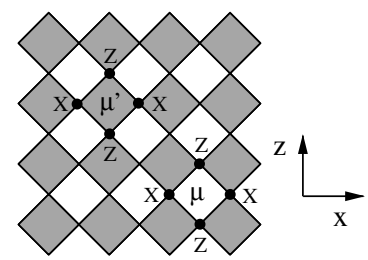

(a)

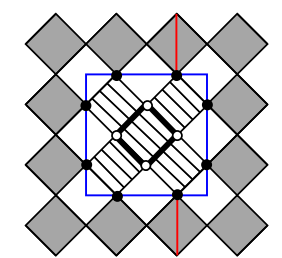

(b)
FIG. 2 (color online). (a) Toric code on a $L \times L$ lattice with $2 L^{2}$ qubits on vertices and periodic boundary conditions in both directions. The Hamiltonian is a sum over white and gray plaquette operators $A_{\mu}=Z_{\mu+\hat{z}} Z_{\mu-\hat{z}} X_{\mu+\hat{x}} X_{\mu-\hat{x}}$. (b) The Hamiltonian in which the hatched plaquettes are omitted has ground space degeneracy of 8 in the topological phase, hence encodes an additional qubit. The logical operators of this qubit are (blue and red) loops encircling the hole. We can call this a white hole qubit as it is obtained by omitting plaquettes in the Hamiltonian, i.e., making a hole in the lattice, which are centered around a white plaquette.

perturbative expansion (see, e.g., [27] for such expansion and further analysis for the honeycomb model), see Supplemental Material [25]. This calculation shows that the ground space degeneracy of the toric code is broken only in $(2 L)$ th-order perturbation theory and we expect that the topological phase would ultimately destabilize via this mechanism.

In order to consolidate this picture, we map our model via a Jordan-Wigner (JW) transformation onto a family of signed transverse field (TF) Ising models on a 2D square lattice, see the details in Supplemental Material [25]. The JW mapping preserves the locality of the interactions, and all reductions are explicit and rigorous. Previous work [28] has shown how to map an Ising gauge theory (assuming open boundary conditions) in a TF (assuming open boundary conditions) by a JW transformation onto the Majorana fermion model discussed here. A standard duality transformation then relates the TF Ising model to the Ising gauge theory in a TF. The mapping clarifies the nature of the topological phase transition and the parameter values for which it will occur. In this analysis we restrict ourselves to the sector $\left\{C_{\mu}^{w}=+1\right\}$. Notice that when $\Delta=0$ the ground state of $H$ is unique since we are in a state of fixed link parity $\left\{V_{i, j}=-1\right\}$. This state lies in the $\left\{C_{\mu}^{g / w}=+1\right\}$ sector since each $C_{\mu}^{g / w}$ is a product of four link operators. As we saw above, at $\lambda \ll \Delta$, the ground space also lies in the $\left\{C_{\mu}^{g / w}=\right.$ $+1\}$ sector. The mapping decouples our fermionic model into a set of TF Ising models $H(\sigma)=-\lambda \sum_{i, j} \sigma_{i, j} S_{i}^{z} S_{j}^{z}-$ $\Delta \sum_{i} S_{i}^{x}$ with the condition $\Pi_{(i, j) \in \text { white } \mu} \sigma_{i, j}=1$ so that the sign of the Ising interactions is determined by gauge bits $\sigma_{i, j} \in\{-1,1\}$ associated with the edges. The gauge condition expresses the fact that the white plaquettes are never frustrated, i.e., $C_{\mu}^{w}=+1$, but a gray plaquette is frustrated when $C_{\mu}^{g}=\Pi_{(i, j) \in \operatorname{gray} \mu} \sigma_{i, j}=-1$. The spectrum of $H(\sigma)$ solely depends on the frustration of the Ising interactions 


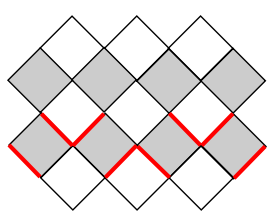

FIG. 3 (color online). The gauge bits $\sigma$ set the Ising interactions to ferromagnetic (FM, black edges) except for an AFM (red bold edges) loop around the torus. This AFM boundary will be felt in the FM phase, but not in the paramagnetic (PM) phase of the model, leading to the topological degeneracy. A loop operator $C_{\gamma}$ in the fermionic model becomes a product of Ising edges which winds around the torus; note that for the depicted sign pattern the loop operator which crosses this domain wall will have -1 eigenvalue.

and the presence of domain walls or homologically nontrivial loops, see Fig. 3. We anticipate the following spectrum, see Fig. 4. At both ends of the parameter region ( $\lambda$ or $\Delta=0$ ), the ground space lies in the unfrustrated TF Ising model sector. We have numerically confirmed this for small lattice sizes for the entire parameter regime. Because of the symmetry between gray and white plaquettes, this finding also motivates the choice for $\left\{C_{\mu}^{w}=+1\right\}$ as the ground sector. Figure 3 depicts a configuration $\sigma$ which represents a homologically nontrivial loop; all plaquettes are unfrustrated, but an Ising model with such an antiferromagnetic (AFM) sign pattern will contain a domain wall of length at least $L$ where bonds are not satisfied. The topological phase is identified as the paramagnetic phase $\left\langle S^{z}\right\rangle=0$ in the TF Ising models. In this phase, the ground spaces of the Hamiltonians $H(\sigma)$ with unfrustrated configurations $\sigma$

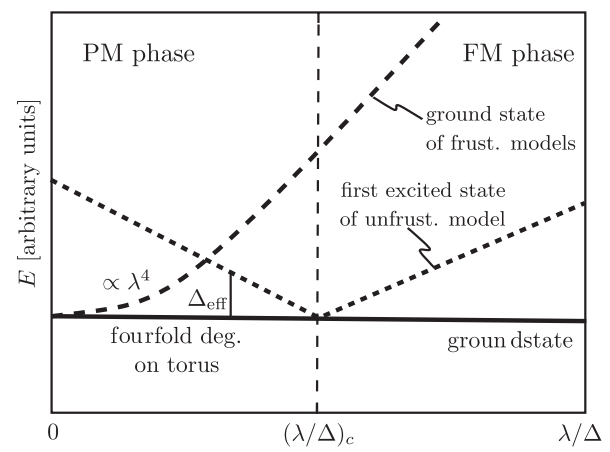

FIG. 4. Sketch of the spectrum of system as a function of $\lambda / \Delta$. For small $\lambda$, the system is in a topological state with a fourfold ground state degeneracy on the torus. The first excited states for small $\lambda$ are Ising models with frustration as determined by the gauge bits. All these models are degenerate for $\lambda=0$; and the degeneracy lifts in fourth-order perturbation theory in $\lambda$, see Eq. (5). The gap of the frustrated model increases monotonically for increasing $\lambda$. The phase transition to a state without topological order happens at the transition point $(\lambda / \Delta)_{c}$ of the unfrustrated Ising model. At this transition the gap of the Ising model closes and the degeneracy of the topological states vanishes. with or without the two nontrivial AFM loops are approximately degenerated. This is the topological degeneracy whose splitting we expect to scale as $\exp (-L / \xi)$, where $\xi$ is the correlation length of the TF ferromagnetic (FM) Ising model. We expect the effective gap $\Delta_{\text {eff }}$ above the degenerate ground space to increase before we reach the secondorder phase transition of the TF FM Ising model, which is known to occur around $\left(\frac{\lambda}{\Delta}\right)_{c} \approx 0.33$ [29]. Elementary excitations of the toric code with $A_{\mu}=+1$ for two gray plaquettes $A_{\mu}$ correspond to ground states of TF Ising models with frustration at those particular gray squares.

The mapping also allows us to study the effect of additional perturbations. One would expect quadratic Majorana fermion perturbations of strength $\epsilon$ on each island due the coupling of the wire ends on an island. If qubits are encoded in wires (as the standard use of Majorana fermions for qubits would prescribe [19]), such couplings lead to energy-level splittings of the encoded qubits of strength $\epsilon$. Here the advantage of our topological encoding becomes clear. Consider a perturbation $U \propto \epsilon i c_{b}^{\mu-\hat{x}} c_{d}^{\mu-\hat{x}}$, where $\mu$ is some white plaquette (by symmetry other perturbations would act similarly) and $\epsilon \ll \lambda$. On the ground space of $H_{0}$, the perturbation acts as a local term $\propto \epsilon X_{\mu-\hat{x}}$; hence, we expect that the topological degeneracy is preserved up to some critical strength $\left(\frac{\epsilon}{\Delta_{\text {eff }}}\right)$, where $\Delta_{\text {eff }}$ is the gap above the degenerate ground space in the topological phase. In practice, we expect these quadratic perturbations to be random (and weak) and hence they could be beneficial in stabilizing the topological quantum memory at finite temperature by limiting the diffusion of anyons (see, e.g., [30]).

We analyze the possibility of implementing the model presented above in superconducting-semiconducting heterostructures. Putting a semiconducting nanowire such as InAs or InSb with strong spin-orbit interaction on top of a conventional superconductor subject to a sufficiently strong magnetic field leads to a pair of Majorana modes located at the ends of the nanowire [12,13]. We focus on a 2D array of superconducting islands each supporting two nanowires leading to four unpaired Majorana modes, see Fig. 1. The product of the four Majorana operators $\mathcal{P}^{i}=$ $c_{a}^{i} c_{b}^{i} c_{c}^{i} c_{d}^{i}$ is fixed by the parity $\mathcal{P}^{i}=(-1)^{n_{i}}$ of the number of electrons $n_{i}$ on the $i$ th island [31,32]. Two Majorana modes $c_{x}^{i}$ and $c_{y}^{j}$ on different superconducting islands $i$ and $j$ interact with each other via the anomalous Josephson interaction

$$
H_{J}=\sum_{i, j} \Gamma_{i, j} V_{i, j} \cos \left[\left(\phi_{j}-\phi_{i}\right) / 2\right]
$$

where $\phi_{i}$ denotes the superconducting phase on the $i$ th island and $\Gamma_{i, j}$ is proportional to the probability amplitude for tunneling a single electron or hole across the link $i, j$ from mode $c_{x}^{i}$ to $c_{y}^{j}$ between the islands $i$ and $j$ [10]. Along the lines of Ref. [22], we shunt each superconducting island with a strong Josephson coupling $E_{J}$ to a common 
ground superconductor. This Josephson coupling fixes the superconducting phases $\phi_{i}$ to a common value due to large fluctuations of the number of electrons (in units of two) on and off the island. Note that this way of freezing out the bosonic degrees of freedom due to the superconductivity is different from the one discussed in Ref. [32] which proposes a large charging energy which fixes the number of electrons by delocalizing the superconducting phase completely. Even though the charge is strongly fluctuating, the fermion parity $\mathcal{P}^{i}$ remains conserved. In the ideal case when all the Josephson couplings are equal $\Gamma_{i, j}=\lambda$, the anomalous Josephson interaction $H_{J}$ implements $V$. Of course, $V$ in general will not have the orientation indicated in Fig. 1, but if we work with a lattice with open boundary conditions (corresponding to the surface code [33]), any sign pattern of the link interactions simply picks out a topological sector with a corresponding pattern of eigenvalues $A_{\mu}= \pm 1$ as the ground space [34], see our discussion in Supplemental Material [25]. From the coding perspective it is well known that topological computation can proceed not only in the trivial syndrome (all eigenvalues of $A_{\mu}=+1$ ) sector but also in some nontrivial syndrome sector.

Next, we discuss the effect of a capacitive coupling

$$
H_{C}=E_{C} \sum_{i}\left(n_{i}-n_{\text {ind }}\right)^{2}
$$

to the ground plate with the capacitive energy $E_{C}=e^{2} / 2 C$, where $C$ is the capacitance of the island with respect to ground and $n_{\text {ind }}$ a constant which is due to gate voltages. For simplicity of notation, we have assumed all the capacitances $C$ and offset charges $n_{\text {ind }}$ to be equal. In the regime with $\lambda \lesssim E_{C} \ll E_{J}$, the capacitive coupling introduces phase slips through the strong Josephson junctions and thus an energy difference between states with different fermion parities. This leads to Eq. (1) with $\Delta \propto E_{C}^{1 / 4} E_{J}^{3 / 4} \cos \left(\pi n_{\text {ind }}\right) e^{-\sqrt{8 E_{J} / E_{C}}}$ [22]. The sign of $\Delta$ depends on the value of $n_{\text {ind }}$ can thus be tuned in principle. A residual interaction between two Majorana modes $c_{x}^{i}$ and $c_{y}^{i}$ with strength $\epsilon$ is due to overlap of the wave functions of the Majorana bound states. However, because the states are localized, this coupling can be made exponentially small by keeping the modes sufficiently far apart from each other. Having sufficiently strong tunneling coupling between the Majorana fermions along the links, a value $\lambda \simeq 200 \mathrm{mK}$ seems realistic as the bare gap of the Majorana wire is likely of the order of a few K [20]. Choosing the Josephson energy to be $E_{J} \simeq 10 \mathrm{~K}$ and a capacitive energy $E_{C} \simeq 5 \mathrm{~K}$, we obtain $E_{J} \gg \Delta \geq \lambda$ as required. The resulting optimal value of $\Delta_{\text {eff }}$ will be of the order of $\lambda \simeq 200 \mathrm{mK}$.

It is possible to tune the tunnel coupling $\Gamma_{i, j}$ by changing the tunneling barrier between islands $i$ and $j$ by a nearby gate. This is an important mechanism through which we can create holes in the lattice. In practice, one can work with a lattice of superconducting islands which represents the surface code with open boundary conditions, encoding one logical qubit. One can apply the ideas of the surface code architecture if (i) one can make (and move) gray and white holes of arbitrary size in this surface and (ii) one can locally measure $X_{i}$ and $Z_{i}$, and prepare $X_{i}$ and $Z_{i}$ eigenstates. The preparation of certain 1-qubit ancillas can then be used to achieve universality [35]. An example of a white hole qubit is depicted in Fig. 2(b). Such a white hole can be obtained by cutting off the four black links surrounding the center white plaquette such that the hatched plaquette terms no longer appear in the effective Hamiltonian. Moving such a white hole could be done by adiabatically changing the strength of Majorana links in order to turn links on and off. The operations (ii) can be implemented using the set up of Fig. 1(b). Instead of a single superconducting island, each site in fact consists of two islands with two Majorana modes each. Most of the time, these islands are coupled to each other via a strong Josephson coupling $E_{J}^{\prime} \gg E_{C}^{\prime}, E_{C}$ (see Fig. 1) such that they essentially act as a single island such that all the discussion above applies unchanged. Increasing the ratio $E_{C}^{\prime} / E_{J}^{\prime}$ turns on a magnetic field along the $z$ axis which can be used to implement rotations around this axis. Additionally, the measurement of $Z_{i}$ can be implemented by coupling one of the superconducting islands to a fermion-parity meter [17]. Single qubit universality is achieved by increasing the overlap of the $a$ and $c$ Majorana modes-by decreasing the length of the topological trivial part of the Majorana wire indicated by the dashed line-and thus effectively implementing a magnetic field along the $x$ axis.

In conclusion, we have proposed a new scheme for topological quantum computation based on encoding in the ground space of a Hamiltonian which emerges from Majorana fermion interactions. It will be worthwhile to further explore the feasibility of this scheme, e.g., the accuracy of the local on-island operations, the precise requirements for adiabatic moving and state preparation of the encoded qubits, the possibility for incorporating error correction, the value $\frac{\lambda}{\Delta}$ at which $\Delta_{\text {eff }}$ is maximal, and the experimental detection of any achieved topological order. We acknowledge fruitful discussions with A. Akhmerov. D.D. V. and F.H. are grateful for support from the Alexander von Humboldt foundation.

[1] A. Yu. Kitaev, Ann. Phys. (N.Y.) 303, 2 (2003).

[2] X.-G. Wen, Phys. Rev. Lett. 90, 016803 (2003).

[3] E. Dennis, A. Kitaev, A. Landahl, and J. Preskill, J. Math. Phys. (N.Y.) 43, 4452 (2002).

[4] R. Raussendorf, J. Harrington, and K. Goyal, New J. Phys. 9, 199 (2007).

[5] H. Bombin and M. A. Martin-Delgado, J. Phys. A 42, 095302 (2009). 
[6] A. G. Fowler, A. M. Stephens, and P. Groszkowski, Phys. Rev. A 80, 052312 (2009).

[7] D. P. DiVincenzo, Phys. Scr. T T137, 014020 (2009).

[8] A. Yu. Kitaev, Ann. Phys. (N.Y.) 321, 2 (2006).

[9] C. G. Brell, S. T. Flammia, S.D. Bartlett, and A.C. Doherty, New J. Phys. 13, 053039 (2011).

[10] A. Yu. Kitaev, Phys. Usp. 44, (Suppl.), 131 (2001).

[11] L. Fu and C. L. Kane, Phys. Rev. Lett. 100, 096407 (2008).

[12] R. M. Lutchyn, J. D. Sau, and S. Das Sarma, Phys. Rev. Lett. 105, 077001 (2010).

[13] Y. Oreg, G. Refael, and F. von Oppen, Phys. Rev. Lett. 105, 177002 (2010).

[14] V. Mourik, K. Zuo, S. M. Frolov, S. R. Plissard, E. P. A. M. Bakkers, and L.P. Kouwenhoven, Science 336, 1003 (2012).

[15] M. T. Deng, C. L. Yu, G. Y. Huang, M. Larsson, P. Caroff, and H. Q. Xu, arXiv:1204.4130.

[16] L.P. Rokhinson, X. Liu, and J. K. Furdyna, arXiv: 1204.4212.

[17] F. Hassler, A. R. Akhmerov, C.-Y. Hou, and C. W. J. Beenakker, New J. Phys. 12, 125002 (2010); F. Hassler, A. R. Akhmerov, and C. W. J. Beenakker, ibid. 13, 095004 (2011).

[18] J. D. Sau, R. M. Lutchyn, S. Tewari, and S. Das Sarma, Phys. Rev. Lett. 104, 040502 (2010).

[19] C. W. J. Beenakker, arXiv:1112.1950.

[20] J. Alicea, Y. Oreg, G. Refael, F. von Oppen, and M. P. A. Fisher, Nature Phys. 7, 412 (2011).

[21] J. D. Sau, D. J. Clarke, and S. Tewari, Phys. Rev. B 84, 094505 (2011).
[22] B. van Heck, A. R. Akhmerov, F. Hassler, M. Burrello, and C. W. J. Beenakker, New J. Phys. 14, 035019 (2012).

[23] B. I. Halperin, Y. Oreg, A. Stern, G. Refael, J. Alicea, and F. von Oppen, Phys. Rev. B 85, 144501 (2012).

[24] S. Gladchenko, D. Olaya, E. Dupont-Ferrier, B. Douçot, L. B. Ioffe, and M. E. Gershenson, Nature Phys. 5, 48 (2008).

[25] See Supplemental Material at http://link.aps.org/ supplemental/10.1103/PhysRevLett.108.260504 for [brief description].

[26] The more familiar representation of the toric code is obtained by unitarily rotating $X \leftrightarrow Z$ on all qubits which are at positions $\nu \pm \hat{x}$ for, say, the gray plaquettes.

[27] J. Vidal, K. P. Schmidt, and S. Dusuel, Phys. Rev. B 78, 245121 (2008).

[28] E. Fradkin, M. Srednicki, and L. Susskind, Phys. Rev. D 21, 2885 (1980).

[29] A. D. B. K. Chakrabarti and P. Sen, Quantum Ising Phases and Transitions in Transverse Ising Models (SpringerVerlag, Berlin, 1996).

[30] J. R. Wootton and J. K. Pachos, Phys. Rev. Lett. 107, 030503 (2011); C. Stark, L. Pollet, A. Imamoğlu, and R. Renneribid., 107030504 (2011).

[31] L. Fu, Phys. Rev. Lett. 104, 056402 (2010).

[32] C. Xu and L. Fu, Phys. Rev. B 81, 134435 (2010).

[33] S. B. Bravyi and A. Yu. Kitaev, arXiv:quant-ph/9811052.

[34] This is not true for the toric code in which plaquette operators are linearly dependent and a flipped sign of a link can lead to frustration.

[35] S. Bravyi and A. Yu. Kitaev, Phys. Rev. A 71, 022316 (2005). 